\title{
Subclinical anxiety and depression are associated with deficits in attentional target facilitation, not distractor inhibition
}

\author{
Pike, Alexandra C. ${ }^{* 1,2}$, Printzlau, Frida A.B. ${ }^{* 3}$, von Lautz, Alexander, $\mathrm{H}^{4}$ \\ Harmer, Catherine J. ${ }^{1,5}$, Stokes, Mark G. ${ }^{3}$, Noonan, MaryAnn P. ${ }^{3}$ \\ ${ }^{1}$ Department of Psychiatry, University of Oxford, Oxford, UK \\ ${ }^{2}$ Institute of Cognitive Neuroscience, University College London, London, UK \\ ${ }^{3}$ Department of Experimental Psychology, University of Oxford, Oxford, UK \\ ${ }^{4}$ Bernstein Center for Computational Neuroscience, Berlin, Germany \\ ${ }^{5}$ Oxford Health NHS Foundation Trust, Warneford Hospital, Oxford \\ *Joint contribution
}

Corresponding author: MaryAnn Noonan, Department of Experimental Psychology, University of Oxford, Anna Watts Building, Radcliffe Observatory Quarter, Woodstock Road, Oxford, OX2 6HG, UK; maryann.noonan@ @sy.ox.ac.uk

Note that the task data we present has been previously analysed in Noonan et al. 2016, Journal of Neuroscience. However, the relationships between these measures and the self-report questionnaire scores that we report here are completely novel.

Word count: 3998

Number of references: 25 


\title{
Subclinical anxiety and depression are associated with deficits in attentional target facilitation, not distractor inhibition
}

\author{
Mood and anxiety disorders are associated with deficits in attentional control \\ involving emotive and non-emotive stimuli. Current theories focus on impaired \\ attentional inhibition of distracting stimuli in producing these deficits. However, \\ standard attention tasks struggle to separate distractor inhibition from target \\ facilitation. Here, we investigate whether distractor inhibition underlies these \\ deficits using neutral stimuli in a behavioural task specifically designed to tease \\ apart these two attentional processes. Healthy participants performed a validated \\ four-location Posner cueing paradigm and completed self-report questionnaires \\ measuring depressive symptoms and trait anxiety. Using regression analyses, we \\ found no relationship between distractor inhibition and mood or anxiety \\ symptoms. However, we find a relationship between target facilitation and both \\ depression and anxiety. Specifically, higher depressive symptoms were \\ associated with reduced target facilitation, and higher anxiety symptoms were \\ associated with enhanced target facilitation in a task-version in which the target \\ location repeated over a block of trials. By contrast, we find the opposite \\ direction of relationships in a task-version in which the location of the \\ forthcoming target was cued on a trial-wise basis. This dissociation may point to \\ separate mechanisms underlying the relationships between depressive and \\ anxiety symptoms and attention and warrants further investigation in clinical \\ populations.
}

Keywords: attention, anxiety, depression, attentional bias, target facilitation

\section{Introduction}

Attention is a core cognitive mechanism for optimising information processing. Failures of attention have been found in mood and anxiety disorders, and recent research has indicated that these failures, in both anxiety (Bishop, 2009; Fox, 1994) and depression (Marazziti et al., 2010; McDermott \& Ebmeier, 2009) may reflect a general deficit in 'cold cognition', rather than one specific to disorder-relevant stimuli (or 'hot cognition'). These failures in attention may be particularly related to difficulties in 
inhibiting distractors (Koster et al., 2005; Yiend \& Mathews, 2001). However, standard tasks often struggle to disentangle inhibitory distractor processing from facilitation of target processing. Here, we use a novel cognitive paradigm, which quantifies and dissociates distractor inhibition from target facilitation. We examine whether these mechanisms are related to self-report trait symptoms of anxiety and depression. Importantly, we use non-emotive stimuli to identify relationships between 'cold' attentional mechanisms and mood symptoms without the potentially confounding salience of disorder-relevant stimuli.

Attentional bias (towards threat-related or negative information) is one of the key cognitive markers in both major depressive disorder and anxiety disorders (BarHaim et al., 2007; Peckham et al., 2010), and may contribute to the development and/or maintenance of these disorders (Disner et al., 2017; Van Bockstaele et al., 2014). Moreover, attentional bias modification, as psychological treatment, might be efficacious in anxiety and depression (Mogoase et al., 2014). Yet, attentional bias is multi-faceted, with distinctions drawn between attentional engagement (initial attentional capture), disengagement (removing attention) and switching (altering the focus of attention between stimuli). Disentangling the component mechanisms is therefore critical in understanding the etiology and developing effective treatments (Clarke et al., 2013).

The classic Posner cueing paradigm disentangles component mechanisms of attentional biases, and studies in subclinical anxious and depressed individuals suggest a selective impairment in disengaging from threat, as opposed to attentional engagement or switching (Koster et al., 2005; Yiend \& Mathews, 2001), but cf. (Koster et al., 2006). However, Mogg et al. (2008) have argued that a general threat-induced slowing in high 
trait anxious individuals challenges the ability of the above studies to properly dissociate the component mechanisms.

More recently, attentional cueing paradigms have dissociated mechanisms underlying attentional facilitation and inhibition (Leber et al., 2016; Noonan et al., 2016). These findings may have implications for the interpretation of deficits of inhibition during attentional disengagement in mood disorders that could alternatively be characterised as a failure to maintain and focus on current goals (target facilitation), particularly in the presence of competition (distractors). Such a hypothesis may account for the general slowing of patients' reaction times (Eysenck et al., 2007; Marazziti et al., 2010). In the present study, we used a validated and internally replicated four location Posner-cueing paradigm that differentiates facilitation and inhibition of mood-neutral stimuli (Noonan et al., 2016). In a large cohort of healthy volunteers, with varied scores on depression and anxiety questionnaires, we tested the degree to which distractor inhibition, as distinct from target facilitation, predicted mood score. We hypothesised a negative relationship between distractor inhibition and both depression and anxiety symptom scores, such that greater symptom levels would reduce the ability of participants to inhibit their responses to distractors. We had no a priori hypotheses about the relationship between target facilitation and these symptom scores.

Our sub-clinical approach is validated by work indicating that anxiety and depression are not categorical disorders (i.e. either present or absent), but operate on a continuum (Helzer et al., 2006). One advantage of such an approach is that it can tease apart factors which precipitate the illness, and therefore potentially relate to development and may offer treatment targets, and factors that maintain the 'state' of being currently unwell (or indeed side effects of the disorder or medication used to treat it). 
To preview our results, we find no evidence that these symptoms are associated with distractor inhibition. However, we do find evidence that impairments in target facilitation may be related to both depressive and anxiety symptoms.

\section{Materials and Methods}

\section{Participants}

Eighty healthy participants were recruited from Oxford and Oxfordshire, UK, of which data is reported from 71 (41 female, for exclusion criteria and participant demographics see supplementary methods). All participants gave informed consent prior to taking part. The Oxford Central University Research Ethics Committee approved the experiment.

\section{Design, stimulus and procedure}

Prior to the main behavioural task participants completed [1] the trait questionnaire of the State Trait Anxiety Inventory (STAI-T; Spielberger, 1983), [2] the Beck Depression Inventory II (BDI-II; Beck et al., 1996) and [3] a questionnaire assessing behaviours associated with cognitive function and mental health, including level of education, family history of mental illness, history of psychotropic drugs, smoking and alcohol consumption (supplementary methods). The task and experimental apparatus have been described in Experiment 2 in Noonan et al. (2016) and are reiterated more fully in the supplementary methods. Briefly, data was collected on Dell laptops with stimulus presentation controlled by MATLAB and supplemented by the Psychophysics Toolbox. After listening to task instructions participants completed two versions of a 4-location Posner cueing paradigm (see figure 1) in a counterbalanced order. Participants made target discrimination judgements, identifying a target either on its own or in the 
presence of a distractor. Targets were triangles or squares and distractors were overlaid triangles and squares. Targets and distractors could appear in any of four quadrants of the screen. The inclusion of 4 possible stimulus locations disentangles target facilitation from distractor inhibition: in the standard 2-location task, a target location cue equally predicts the distractor location (and vice versa). Participants discriminated between the two target types through mouse button responses. They were instructed to do so as quickly and accurately as possible and received auditory accuracy feedback.

Both task versions contained three block types that differed in terms of which stimulus was predictable. A cue predicted either the [1] target location (target condition), [2] distractor location (distractor condition), or [3] was not spatially predictive of either stimulus (neutral condition). In one version of the task the cued stimulus locations were flexible and varied on a trial wise basis. On every trial of this flexible version of the task, the cue, a small white dot, was presented to one corner of the fixation cross for $100 \mathrm{~ms}$. In another version of the task the cued stimulus was fixed for each block of trials. In this blocked version of the task, the cue appeared at the start of each block only, and, in the case of target or distractor cues, predicted the location of that stimulus throughout the block. The neutral condition in the blocked version was equivalent to the flexible version in that no predictive information was provided. These manipulations allowed us to probe target facilitation and distractor suppression as two dissociable mechanisms. Finally, distractor presence was also manipulated in a blockwise manner. This allowed us to differentiate between the benefit of a distractor cue that cues a distractor; and the benefit of a distractor cue that simply reduced the spatial uncertainty of the target location.

From these six different cuing conditions we focused on two a priori measures: target facilitation with distractor interference (reaction time (RT) difference between 
neutral cuing and target cuing in the presence of a distractor) and distractor inhibition with the distractor present (difference between neutral and distractor cuing in the presence of a distractor).

\section{[Figure 1]}

\section{Data analyses}

All analyses were performed in MATLAB (various versions) (Mathworks), and R (version 3.5.1). Median RTs derived from the tasks (see supplementary methods for outlier removal) were used as the dependent variable in a series of multiple regression analyses, median group split 3-way mixed ANOVAs, and independent samples t-tests.

Multiple regression was performed using a stepwise procedure. A 'null' model was constructed and included nuisance covariates: the mean of the median RTs across the task versions and cuing conditions, the relevant cuing effect for the other task version (in order to remove shared variance between task versions), age, gender, number of years of education completed, medication, family history of mental health problems, alcohol intake per week and cigarette use per week. The experimental model included the mean-centred STAI-T and BDI-II scores as well as the covariates from the null model. Comparison of model fits then examined the effects of mood scores controlling for nuisance variables. As the models did not suffer from multicollinearity (defined as tolerance $>0.1$, and variance inflation factor $<10$ ) we used STAI-T and BDIII scores in the same regression model.

\section{Bayes Factor analyses}

We complement the classical frequentist statistics with Bayes Factor analyses (see supplementary methods). These supporting analyses indicate the strength of evidence in 
favour of the null hypothesis $\mathrm{H}_{0}$ compared to $\mathrm{H}_{1}$ (reported here as $B F_{01}$ ) and the strength of the evidence for the model of interest (reported as $B F_{10}$ ).

\section{Results}

\section{Demographics and questionnaires}

The distribution of BDI-II and STAI-T scores are presented in supplementary figure 2, with the STAI-T scores comparable to the published norms $(\mathrm{M}=36, \mathrm{SD}=10$; (Spielberger, 1983)). BDI-II scores ranged from 0-27 $(\mathrm{M}=6.2, \mathrm{SD}=6.6)$. All means and standard deviations of key measures of interests (BDI-II and STAI-T score, and covariates) are reported in the supplementary results.

\section{Attention task}

Analysis of the impact of cue type and distractor presence on RT are reported in Noonan et al., (2016) (Experiment 2). For completeness, we present a summary of these results in the supplementary material.

For the current experiment, we examined these attentional effects as a function of trait STAI-T scores and BDI-II scores.

\section{BDI-II and STAI-T predict target facilitation but not distractor inhibition}

Using multiple regression analyses, we examined the degree to which non-clinical anxiety and depression symptoms related to two key a priori cueing effects: distractor inhibition (TDn-TDt), or target facilitation (TDn-TDt) in either flexible or blocked cueing task versions. 
Contrary to our hypotheses, anxiety and depression scores were not predictive of distractor inhibition effects, with neither BDI-II nor STAI-T scores explaining a significant amount of variance within either version of the task, no significant improvement over the null model by adding BDI-II or STAI-T, and the resulting models being non-significant in both the blocked version (overall model: $R^{2}=0.08, F_{11,59}=0.48$, $\left.p=0.91, B F_{01}=1.84\right)$, and flexible version of the task $\left(R^{2}=0.20, F_{11,59}=1.35, p=0.223\right.$, $\left.B F_{01}=1.59\right)$.

By contrast, BDI-II $(\beta=-0.0027, t=-3.34, p=0.0015)$, and STAI-T scores $(\beta=$ $0.001, t=2.09, p=0.041$ ), explained a significant amount of variance in target enhancement effects for the blocked task version. The findings suggest that more depressive symptoms related to reduced target facilitation, whereas higher anxiety symptom levels were associated with increased target facilitation. The overall model was significant $\left(R^{2}=0.276, F_{11,59}=2.05, p=0.039\right)$, and the model significantly improved with the addition of our variables of interest $\left(\Delta R^{2}=0.145, \Delta F_{2,59}=5.93, p=\right.$ $\left.0.0045, B F_{10}=20.8\right)$. Secondly, we find that BDI-II $(\beta=0.0019, t=2.29, p=0.026)$ and STAI-T scores $(\beta=-0.0012, t=-2.57, p=0.013)$ explain a significant amount of variance in target facilitation in the flexible version of the task. The direction of these effects is opposite compared to the blocked task version: the results indicate that more depressive symptoms related to increased target facilitation, whereas higher anxiety symptom levels as associated with reduced target facilitation. The overall model was significant $\left(R^{2}=0.299, F_{11,59}=2.28, p=0.021\right)$, and the addition of STAI-T and BDI-II variables significantly improved prediction over a null model including covariates, $\left(\Delta R^{2}\right.$ $\left.=0.081, \Delta F_{2,59}=3.41, p=0.040, B F_{10}=3.10\right)$. Notably, in both analyses, the presence of a distractor is essential for this effect (Tn-Tt $p \mathrm{~s}>0.5$ for STAI-T and BDI-II predictors). 
[Figure 2]

For completeness, we performed a median split on both BDI and STAI-T scores and examined distractor inhibition and target facilitation in the high and low score participants. Independent samples t-tests complemented the regression analyses, with no significant effects of BDI-II or STAI-T on distractor inhibition in either task version, but a significant effect of BDI-II on target facilitation in the blocked task $\left(t_{68.4}=-2.22\right.$, $p=0.03, B F_{10}=1.95 ;$ Figure $\left.2 \mathrm{c}\right)$ and an effect of trait anxiety on target facilitation in the flexible task $\left(t_{67.7}=-2.1547, p=.03474, B F_{10}=1.7317\right.$; Figure $\left.2 d\right)$. However, Bayes Factors indicated only anecdotal evidence, and the double dissociation observed above was not present. Means and standard deviations are reported in supplementary results: cuing effects by median-split STAI-T and BDI-II scores.

\section{Mixed model analysis of condition differences in RT and STAI-T/BDI-II scores}

Finally to rule out a confounding relationship between general RT slowing and higher mood symptoms (Eysenck et al., 2007; Marazziti et al., 2010) we performed two multiple regression analyses on RTs across each condition, for each version of the task, covarying out demographic information described above. Neither STAI-T nor BDI-II scores explained a significant amount of variance when added to a null model containing covariates in the blocked $\left(\Delta R^{2}=0.0058, \Delta F_{2,61}=0.21, p=0.815, B F_{01}=3.3\right)$, or flexible version of the task $\left(\Delta R^{2}=0.0035, \Delta F_{2,61}=.12, p=0.886, B F_{01}=3.8\right)$.

\section{Discussion}

Target facilitation and inhibition are governed by distinct neurocognitive mechanisms (Noonan et al., 2016). Here we explored the implications of this for understanding deficits of selective attention in mood disorders. Using an adapted mood-neutral Posner 
cueing task that differentiates top-down and bottom-up mechanisms of target facilitation and distractor inhibition in a sub-clinical population, we examined the degree to which individual differences in depression and anxiety symptomology were specifically associated with distractor inhibition. Contrary to our hypotheses, we found no evidence that subclinical depression or anxiety scores were associated with a reduced capacity to inhibit distractors. However, we did find evidence for a complex relationship between target facilitation and both anxiety and depression scores, with an unpredicted double dissociation in the direction of the effects between task-versions.

Specifically, in the flexible version of the task, which is likely to rely on working memory-based top-down control (Noonan et al., 2016), individuals with high trait anxiety had reduced target facilitation effects. By contrast, individuals with high depressive symptom levels showed increased target facilitation effects and were more able to utilise preparatory cues to orientate attention towards a target. In the blocked task-version, which may require a combination of implicit mechanisms of predictive coding (Friston, 2010), and elements of top-down control, we find the reverse pattern. We showed individuals with higher depression symptomatology had reduced target facilitation effects in the blocked task. By contrast, higher trait anxiety scores were associated with increased target facilitation effects, with individuals better able to identify a target when its location becomes increasingly predictable. Our effects were only present when distractors were competing for attentional resources, suggesting that anxiety and depression may impair goal-directed processing in the presence of distracting information.

While our task cannot fully orthogonalise effects of top-down and implicit mechanisms of target facilitation, some of the results are congruent with past literature. Consistent with individuals with high depressive symptomatology being less able to 
benefit from an increasingly predictable target location, depression has previously been linked to deficits in predictive coding (Badcock et al., 2017). By contrast, our participants with high trait anxiety may be less able to use flexible top-down cues to facilitate target discrimination in this task because of underlying deficits in workingmemory (Vytal et al., 2016) or top-down control, perhaps linked to reduced prefrontal activity (Bishop, 2009). However, previous research does not offer explanations for better working memory/top-down control to accompany increased depressive symptoms, or improved predictive coding in high trait anxiety individuals.

Past studies that report general deficits in attentional control using neutral stimuli in subclinical anxiety (Bishop, 2009; Eysenck et al., 2007; Fox, 1994) and depression (McDermott \& Ebmeier, 2009) often interpreted them as failures of inhibition. For example, negative priming (i.e. slowed responses to a location or item that was just ignored) is reduced in individuals with high trait anxiety (Fox, 1994) and depression (MacQueen et al., 2000). However, methodological challenges may limit the negative priming paradigm as a measure of inhibition, as the effect could also be related to feature and/or response conflict from previous trials (Macleod et al., 2003). Using the Eriksen-Flanker response-conflict task, Bishop (2009) found that high trait anxious individuals showed slowed performance accompanied by attenuated recruitment of the prefrontal cortex. In light of reduced target facilitation in our study the response-conflict induced slowing in previous tasks could reflect an impairment in the target maintaining its goal-relevant status in the presence of distractors, rather than distractor inhibition deficits. Finally, whether cognitive control deficits in trait anxiety and depression are ameliorated when attentional resources are fully occupied is unresolved (Bishop, 2009). Our findings of reduced target facilitation in high anxious/depressed individuals only when distractors were competing for attentional resources are in line with predictions 
from Attentional Control Theory (Eysenck et al., 2007). Future studies should ensure cognitive and perceptual load is controlled for to further unravel their impact on attentional control within anxiety and depression.

Our previous work disentangled mechanisms of facilitation and inhibition (Noonan et al., 2016). Here we demonstrate the clinical relevance of this for understanding attentional control deficits in mood disorders. If distractor inhibition is not as flexible as top-down control and less reliant on working memory, this will have implications for the cognitive models of anxiety and depression (e.g. Attentional Control Theory) that have assumed deficits in top-down, working-memory dependent, inhibitory control (Eysenck et al., 2007). Moreover, with cognitive treatments for anxiety and depression, such as attentional bias modification with emotional stimuli, gaining traction in recent years (Mogoase et al., 2014), findings of general deficits in target facilitation may suggest treatment could focus on general goal-orientated information-processing.

Limitations: While the effects reported are significant, Bayes analyses generally provide only anecdotal evidence for the tested hypotheses. Further replication is therefore essential. Experiments including mood-relevant stimuli could also be performed, to examine whether the relationships we have found here might contribute to attentional biases toward negative or emotional stimuli. Finally, the unexpected double dissociation in target facilitation between different task versions and symptom measures warrants further replication in tasks specifically designed to separate top-down and implicit mechanisms of target facilitation.

To conclude, we suggest that trait anxiety and depressive symptomology are related to general deficits in attentional control. This deficit does not appear to be driven by distractor inhibition difficulties as we had expected, but by impairments in goal- 
directed information processing (target facilitation) in the presence of competing information. Our study highlights the importance in disentangling top-down and implicit contributions to faciliatory and inhibitory information-processing mechanisms to better understand the etiology and maintenance of anxiety and depression disorders.

\section{Acknowledgements}

Supported by a Medical Research Council (MRC) career development award to MGS. The research was also supported by the National Institute for Health Research (NIHR) Oxford Health Biomedical Research Centre Programme. The views expressed are those of the authors and not necessarily those of the NHS, the NIHR or the Department of Health. We would also like to thank Philip Noonan for technical assistance.

\section{Declaration of interest statement}

The authors declare no conflicts of interest.

\section{References}

Badcock, P. B., Davey, C. G., Whittle, S., Allen, N. B., \& Friston, K. J. (2017). The Depressed Brain : An Evolutionary Systems Theory. Trends in Cognitive Sciences, 21(3), 182-194. http://doi.org/10.1016/j.tics.2017.01.005

Bar-Haim, Y., Lamy, D., Pergamin, L., Bakermans-Kranenburg, M. J., \& van IJzendoorn, M. H. (2007). Threat-related attentional bias in anxious and nonanxious individuals: a meta-analytic study. Psychological Bulletin, 133(1), 124. http://doi.org/10.1037/0033-2909.133.1.1

Beck, A., Steer, R., \& Brown, G. (1996). Beck Depression Inventory -II. San Antonio: TX.

Bishop, S. J. (2009). Trait anxiety and impoverished prefrontal control of attention. Nature Neuroscience, 12(1), 92-98. http://doi.org/10.1038/nn.2242

Clarke, P. J. F., Macleod, C., \& Guastella, A. J. (2013). Assessing the role of spatial 
engagement and disengagement of attention in anxiety-linked attentional bias : a critique of current paradigms and suggestions for future research directions. Anxiety, Stress \& Coping, 26(1), 1-19. http://doi.org/10.1080/10615806.2011.638054

Disner, S. G., Shumake, J. D., Beevers, C. G., Disner, S. G., Shumake, J. D., \& Self-, C. G. B. (2017). Self-referential schemas and attentional bias predict severity and naturalistic course of depression symptoms naturalistic course of depression symptoms, 9931. http://doi.org/10.1080/02699931.2016.1146123

Eysenck, M., Derakshan, N., Santos, R., \& Calvo, M. (2007). Anxiety and cognitive performance: attentional control theory. Emotion, 7(2), 336-53. http://doi.org/10.1037/1528-3542.7.2.336

Fox, E. (1994). Attentional bias in anxiety: A defective inhibition hypothesis. Cognition \& Emotion, 8(2), 165-195. http://doi.org/10.1080/02699939408408934

Friston, K. (2010). The free-energy principle: a unified brain theory?, (January), 1-12. http://doi.org/10.1038/nrn2787

Helzer, J., Kraemer, H., \& Krueger, R. (2006). The feasibility and need for dimensional psychiatric diagnoses. Psychological Medicine, 36(12), 1671-1680.

Koster, E. H. W., Crombez, G., Verschuere, B., Damme, S. Van, \& Wiersema, J. R. (2006). Components of attentional bias to threat in high trait anxiety: Facilitated engagement, impaired disengagement, and attentional avoidance. Behaviour Research and Therapy, 44, 1757-1771. http://doi.org/10.1016/j.brat.2005.12.011

Koster, E. H. W., Raedt, R. De, Goeleven, E., Franck, E., \& Crombez, G. (2005). Mood-Congruent Attentional Bias in Dysphoria : Maintained Attention to and Impaired Disengagement From Negative Information, 5(4), 446-455. http://doi.org/10.1037/1528-3542.5.4.446

Leber, A. B., Gwinn, R. E., Hong, Y., \& Toole, R. J. O. (2016). Implicitly learned suppression of irrelevant spatial locations. Psychonomic Bulletin \& Review, 23, 
1873-1881. http://doi.org/10.3758/s13423-016-1065-y

Macleod, C. M., Dodd, M. D., Sheard, E. D., Wilson, D. E., \& Bibi, U. (2003). In Opposition to Inhibition. In B. H. Ross (Ed.), The Psychology of Learning and Motivation (Vol. 43).

MacQueen, G. M., Tipper, S. P., Young, L. T., Joffe, R. T., \& Levitt, A. J. (2000). Impaired distractor inhibition on a selective attention task in unmedicated, depressed subjects. Psychological Medicine, 30, 557-564.

Marazziti, D., Consoli, G., Picchetti, M., Carlini, M., \& Faravelli, L. (2010). Cognitive impairment in major depression. European Journal of Pharmacology, 626(1), 8386. http://doi.org/10.1016/j.ejphar.2009.08.046

McDermott, L. M., \& Ebmeier, K. P. (2009). A meta-analysis of depression severity and cognitive function. Journal of Affective Disorders, 119(1-3), 1-8. http://doi.org/10.1016/j.jad.2009.04.022

Mogg, K., Holmes, A., Garner, M., \& Bradley, B. P. (2008). Effects of threat cues on attentional shifting, disengagement and response slowing in anxious individuals. Behaviour Research and Therapy, 46(5), 656-667. http://doi.org/10.1016/j.brat.2008.02.011

Mogoase, C., David, D., \& Koster, E. H. W. (2014). Clinical Efficacy of Attentional Bias Modification Procedures: An Updated Meta-Analysis. Journal of Clinical Psychology, 70(12), 1133-1157.

Noonan, M. P., Adamian, N., Pike, A., Printzlau, F., Crittenden, B. M., \& Stokes, M. G. (2016). Distinct Mechanisms for Distractor Suppression and Target Facilitation. Journal of Neuroscience, 36(6), 1797-1807. http://doi.org/10.1523/JNEUROSCI.2133-15.2016

Peckham, A. D., McHugh, R. K., \& Otto, M. W. (2010). A meta-analysis of the magnitude of biased attention in depression. Depression and Anxiety, 27(12), 1135-1142. http://doi.org/10.1002/da.20755 
Spielberger, C. D. (1983). Manual for the state-trait anxiety inventory (form V). Palo Alto, CA: Consulting Psychologists Press.

Van Bockstaele, B., Verschuere, B., Tobboel, H., De Houwer, J., Crombez, G., \& Koster, E. (2014). A review of current evidence for the causal impact of attentional bias on fear and anxiety. Psychological Bulletin, 140(3), 682-721.

Vytal, K. E., Arkin, N. E., Overstreet, C., Lieberman, L., \& Grillon, C. (2016). Inducedanxiety differentially disrupts working memory in generalized anxiety disorder. BMC Psychiatry, 1-9. http://doi.org/10.1186/s12888-016-0748-2

Yiend, J., \& Mathews, A. (2001). Anxiety and attention to threatening pictures. The Quarterly Journal of Experimental Psychology A, 54A(3), 665-681. http://doi.org/10.1080/713755991 
Figure 1

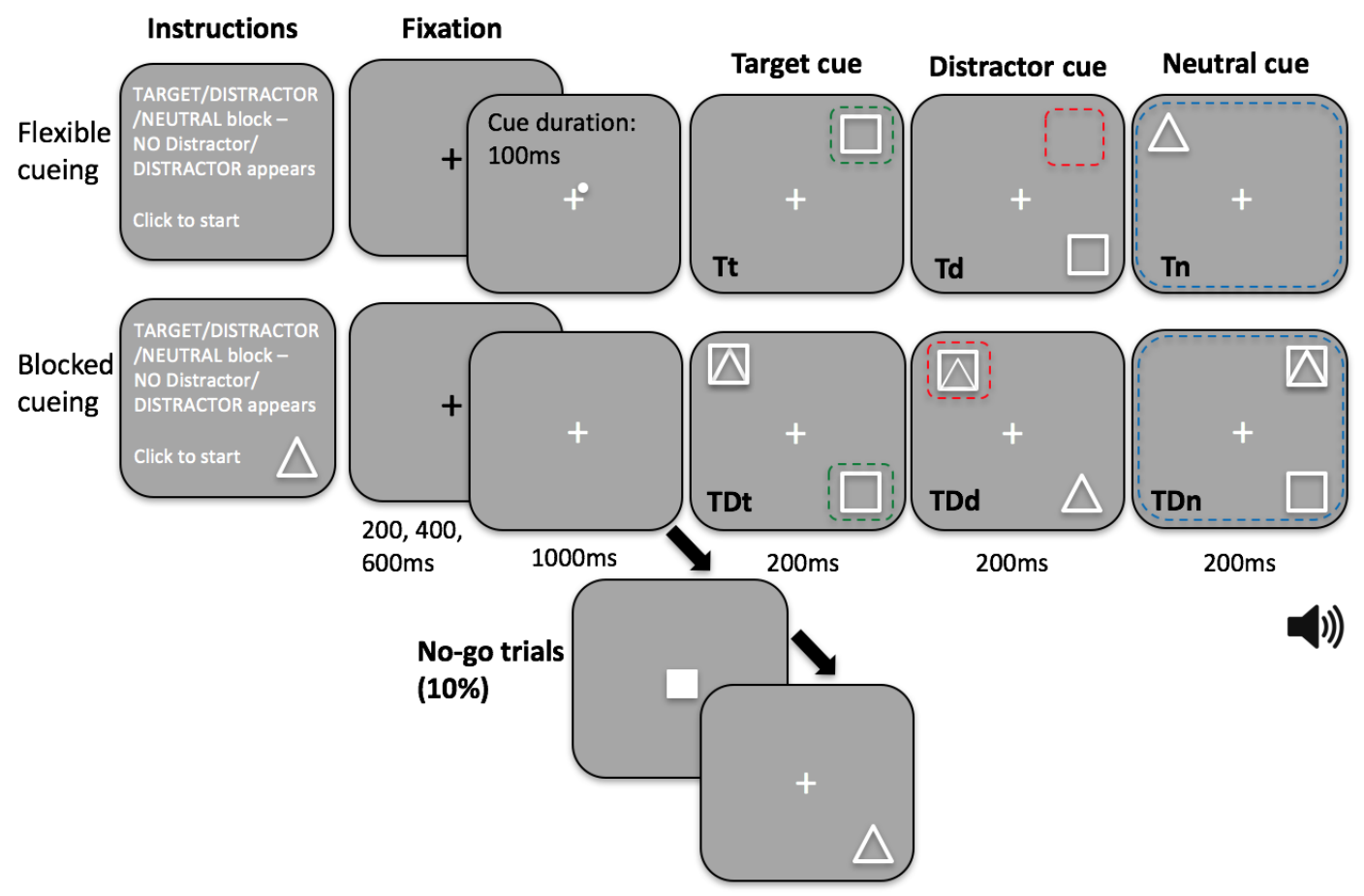




\title{
Figure 2
}

a
BDI-II score
STAI-T score
Overall RT

Flexible distractor inhibition

Blocked distractor inhibition

$$
\text { Age }
$$

Years of education

Units of alcohol per week

Cigarettes per week

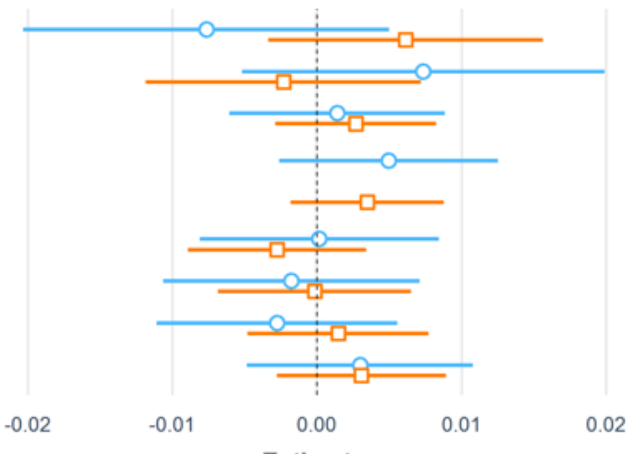

Estimate
Model

- - Blocked task version, distractor inhibition

$-\square-$ Flexible task version, distractor inhibition

b
BDI-II score
STAI-T score
Overall RT

Flexible target facilitation Blocked target facilitation

$$
\text { Age }
$$

Years of education

Units of alcohol per week

Cigarettes per week
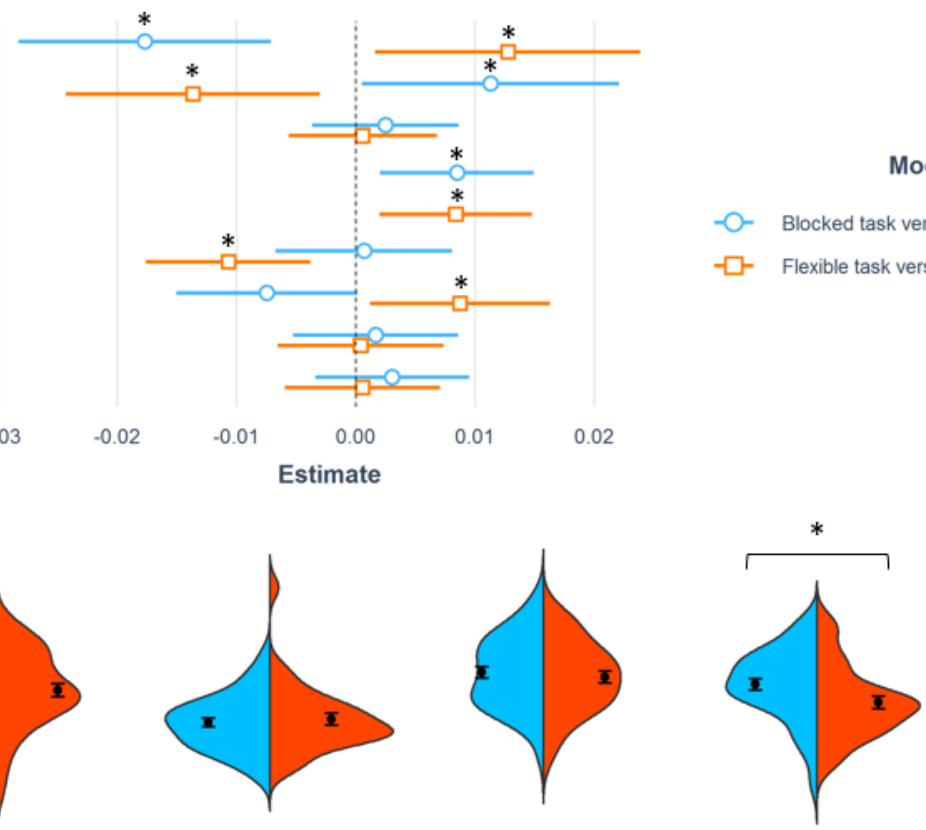

STAI-T

- Blocked task version, target facilitation

$-\square-\quad$ Flexible task version, target facilitation

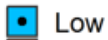

- High

$$
\text { Flexible }
$$

\section{Cuing effect}
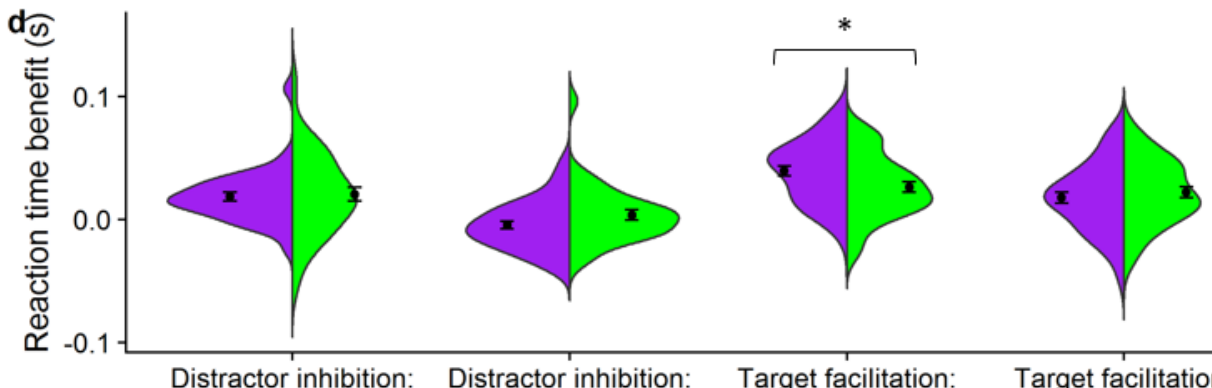

BDI-II

- Low

High

\begin{abstract}
Flexible
\end{abstract}

\section{Cuing effect}




\section{Figure captions}

Figure 1: Task schematic. Participants performed two versions of an adapted fourlocation Posner cuing task. In both task versions participants were instructed whether the forthcoming block would be a target, distractor or neutral cue condition. They were also informed whether a distractor would be present or not. In the blocked cueing task the participants were also informed of the location of the cued stimulus (target or distractor) which was valid for the block duration. Each trial began with a fixation cross (jittered: 200/400/600 ms), which would turn from black to white 1000ms before stimulus onset. In the flexible cueing task a spatially predictive cue was presented in the corner of the white fixation cross for 100ms. In neutral cue blocks the cue was presented randomly and did not provide predictive information. The dashed coloured squares represent the spatially cued location (at the bottom right for illustration purposes only) and illustrate how participants should optimally distribute their attention in the three conditions. Targets were squares or triangles and distractors were superimposed squares and triangles. Accuracy feedback was provided with an auditory tone at the end of each trial. The three cue conditions and two distractor conditions resulted in six block types for each version of the task: [1] Target cuing, target present, no distractor (Tt), [2] Distractor cuing condition, target present, no distractor (Td), [3] Neutral cuing condition, target present, no distractor (Tn), [4] Target cuing condition, target and distractor present (TDt), [5] Distractor cuing condition, target and distractor present (TDd) and [6] Neutral cuing condition, target and distractor present (TDn). The six conditions in each task would occur in randomised order an equal number of trials per block.

Figure 2. Plots of the estimated regression coefficients (and confidence intervals) for models predicting [a] target facilitation and [b] distractor inhibition effects on RT in each version of the task. No significant predictors of distractor inhibition were found. There is a significant negative effect of BDI-II score on blocked target facilitation; a significant positive effect of BDI-II score on flexible target facilitation; a significant negative effect of STAI-T score on flexible target facilitation; and a significant positive effect of STAI-T on blocked target facilitation. There are also relationships between some nuisance covariates such as age and years of education on flexible target 
facilitation. The estimates of the regression coefficients have been converted to the same numeric scale. Stars indicate significant regression coefficients. Violin plots, with overlaid box plots, show the distribution and probability density of the median RT benefits for each participant for the Blocked and Flexible versions of the task, divided into groups with above-median and below-median STAI-T [c], and above-median and below-median BDI [d], for the two different cueing effects examined in this paper. Stars indicate a significant t-test on the cuing effect by median-split questionnaire data. Error bars indicate \pm 1 SEM. 